\title{
II Tolvaptan negato. L'impegno di AIRP contro la decisione dell'Aifa
}

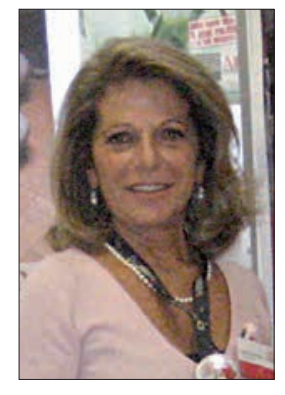

Luisa Sternfeld Pavia
Cari amici,

notizie buone e meno buone in questa primavera 2016, che sboccia mentre vi scrivo queste righe.

Le notizie buone sono quelle che riguardano le nostre attività, sempre intense e di costante impegno.

AIRP è stata presente alla Giornata Mondiale del Rene, che si è celebrata il 10 Marzo in tutto il mondo e che, in questa edizione, era dedicata alle malattie renali nei bambini.

Per l'occasione, siamo stati presenti con postazioni dedicate a Milano (Ospedale San Raffaele), a Treviso (Ospedale Ca' Foncello) e a Salerno, presso I'Università degli Studi. I nostri desk hanno diffuso informazioni sul rene policistico autosomico dominante, ADPKD, e sulla malattia policistica renale autosomica recessiva infantile, ARPKD.

Inoltre, AIRP ha collaborato con SINePe (Società Italiana di Nefrologia Pediatrica) e con altre Associazioni, per produrre un video destinato alla prevenzione, intitolato "Custodisci il tuo rene" (potete vederlo sul sito www.renepolicistico.it).

E, ancora, stiamo sostenendo la raccolta fondi per un progetto di Studio sul registro di ARPKD presso la Clinica De Marchi di Milano, Unità di Nefrologia e Dialisi Pediatrica, diretta dal Prof. Giovanni Montini, in memoria della piccola Beatrice.

\#RicordandoBeatricexdireARPKDstop

Notizie meno buone, invece, sul fronte delle istituzioni. L'Agenzia Italiana del Farmaco (Aifa) ha, infatti, negato la rimborsabilità del farmaco Tolvaptan, inserendolo in classe $\mathrm{C}$, cioè a totale carico del paziente. II costo della terapia non è sostenibile per la maggior parte dei pazienti e delle loro famiglie, che, di conseguenza, non vi potranno accedere.

AIRP ha denunciato la grave ingiustizia e la totale assenza di conoscenza della gravità della malattia, oltre all'indifferenza delle autorità verso la sofferenza di questi pazienti. La voce dei pazienti di tutta Italia esprime la rabbia e il dolore delle famiglie lasciate sole dalle istituzioni.

Accepted: May 4, 2016

Published online: May 30, 2016

Indirizzo per la corrispondenza:

Dr.ssa Luisa Sternfeld Pavia

AIRP Associazione Italiana Rene Policistico onlus

Via A. Bazzini 2

20131 Milano

luisa.sternfeld.airp@renepolicistico.it
"Dobbiamo far conoscere alla gente il problema dei malati di rene policistico come me, la mia famiglia (tre figli malati) e tutti gli altri. Non si può far pagare un farmaco che permette a chi lo assume di salvargli la vita", scrive C.M. una paziente affetta da rene policistico, 54 anni, di Arese, Milano. "lo lo sto già assumendo, perché faccio parte del protocollo al Policlinico di Milano, ma non voglio ritenermi una dei pochi fortunati. Deve essere per tutti".

Con la sua decisione, Aifa dimostra di considerare di serie B i pazienti affetti da rene policistico, ai quali è negata l'unica cura in grado, oggi, di dare una nuova speranza di vita più dignitosa e un miglioramento delle condizioni di salute.

Tolvaptan è, infatti, il primo trattamento in grado di rallentare la crescita delle cisti, preservando la funzionalità renale. AIRP chiede, quindi, che una decisione cos̀ ingiusta venga prontamente modificata ed è impegnata a sollevare il problema presso le massime autorità di Governo, Parlamento e Ministeri competenti.

Desidero ringraziare tutti voi, infine, per la preziosa generosità che dimostrate, decidendo di devolvere il vostro $5 \times 1000$ alla ricerca sul Rene Policistico.

Se, in futuro, le vite di quanti oggi soffrono potranno cambiare in meglio, sarà in gran parte merito vostro.

Tra le grandi conquiste del 2015, rese possibili dalle vostre donazioni, c'è stato l'avvio del trial sull'uomo presso l'IRCCS Ospedale San Raffaele sull'efficacia della molecola 2-deossi-glucosio (2DG), nella regressione e nella riduzione delle cisti renali. La Dr.ssa Alessandra Boletta e la sua equipe hanno ottenuto ottimi risultati e siamo molto felici di poter annunciare che, grazie alle vostre donazioni, AIRP aumenterà il suo sostegno alla ricerca sulla molecola 2DG per l'anno 2016.

Il secondo importante traguardo che ci avete permesso di raggiungere è il test genetico per l'analisi dei geni PKD1 e PKD2 mediante Next Generation Sequencing (NGS) con la piattaforma IonTorrent Personal Genome Machine (PGM), che è, oggi, possibile effettuare nel laboratorio di Genetica Medica degli Spedali Civili di Brescia: una vera a propria conquista nella qualità della vita dei pazienti e in quella delle loro famiglie.

Tengo fortemente a sottolineare che, senza il vostro aiuto, nulla di tutto ciò avrebbe potuto realizzarsi.

Salutandovi, rinnovo l'invito a donare ad AIRP per sostenere insieme la ricerca sul Rene Policistico e dare così, ogni giorno, nuove speranze a chi lotta contro gli effetti di questa patologia.

Diciamo $5 \times 1000$ volte basta al \#RenePolicistico Un caro saluto a tutti! 